\title{
Peripapillary retinal blood flow in normal tension glaucoma
}

\author{
Hak Sung Chung, Alon Harris, Larry Kagemann, Bruce Martin
}

\begin{abstract}
Aims-To determine if normal tension glaucoma (NTG) patients differ from age matched controls in blood flow to the peripapillary retina, as measured with confocal scanning laser Doppler flowmetry (cSLDF; "Heidelberg retinal flowmetry"). Methods-12 NTG patients and 12 age matched controls were compared using (a) $10 \times 10$ pixel boxes (the instrument default sample size), taken from the nasal and temporal peripapillary retina, (b) the average from two of these boxes, and (c) every qualifying pixel within the peripapillary retina.

Results-Patients and controls did not differ in blood flow measured using the default sample from a single $10 \times 10$ pixel box, placed in either the temporal or nasal peripapillary retina, or expressed as the average from these two boxes. However, in histograms using every pixel from the peripapillary retina, NTG patients displayed significantly higher percentages of minimal flow pixels (defined as less than one arbitrary unit of flow: $30 \% v 19 \%$, p $<0.01$ ), and significantly lower flow in the 25 th, 50th, and 75 th percentile flow pixel (each $p<0.05)$ than did age matched controls.

Conclusion-NTG is characterised by reduced blood flow in the peripapillary retina, a result suggesting that blood flow deficits accompany, and perhaps may contribute to, disease development in these patients.

(Br F Ophthalmol 1999;83:466-469)
\end{abstract}

Department of

Physiology and

Biophysics, Indiana

University School of

Medicine,

Indianapolis, Indiana,

USA

H S Chung

A Harris

L Kagemann

Medical Sciences

Program, Indiana

University School of

Medicine,

Bloomington, Indiana,

USA

B Martin

Correspondence to:

Alon Harris, $\mathrm{PhD}$

Department of

Ophthalmology, Rotary 134, Indiana University School of Medicine, Indianapolis, IN 4602-6195, USA.

Accepted for publication 27 October 1998 illary region ${ }^{7}$ throughout the brain. ${ }^{8}$ However, these haemodynamic markers provide no direct infor- mation about perfusion of the retina or optic nerve head. In this study, confocal scanning laser Doppler flowmetry (cSLDF) was used to determine capillary blood flow in the peripapillary retina near the optic nerve head. ${ }^{9}$ Previous studies that have used this technique have failed to detect a perfusion difference between controls and patients with NTG. ${ }^{10}{ }^{11}$ However, those experiments utilised the default $10 \times 10$ pixel sampling box, a method that exhibits a high coefficient of variation when repeated on a weekly basis. ${ }^{12}$ In this study, in addition to conventional analysis, we utilised a new methodology that includes every qualifying pixel within the entire cSLDF image. ${ }^{12}$ Besides measuring the distribution of low and high flow pixels within the sample, this novel method reduces the coefficient of variation of repeated measurements by nearly $50 \%{ }^{12}$

\section{Patients and methods}

SUBJECTS

Twelve patients (seven women, five men; mean age 54 (SD 3) years) and 12 age and sex matched controls with normal eye examinations (eight women, four men; mean age 49 (3) years) were recruited for study. A homogeneous group of patients with early stage normal tension glaucoma were recruited from the Indiana University Hospital Glaucoma Service. All patients had either substantial optic disc cupping $(\mathrm{C} / \mathrm{D}$ ratio $\geqslant 0.8)$, or a combination of less severe cupping (C/D ratio 0.6 to 0.7 ) and visual field defects measured as either mean deviation (MD) or corrected pattern standard deviation (CPSD). For the group of 12 patients, C/D ratio averaged 0.7 (SD 0.1), MD averaged $4.2(4.9) \mathrm{dB}$, and CSPD averaged 6.37 (5.44) for the central 24-2 of Humphrey automated perimetry. All subjects had intraocular pressure less than $21 \mathrm{~mm} \mathrm{Hg}$ (mean IOP 16 (2) $\mathrm{mm} \mathrm{Hg}$ ), with normal diurnal curve measurements. Gonioscopy indicated open anterior chamber angles in all patients, and none had a history of orbital or ocular trauma. Both patients and controls were free from any history of hypertension or diabetes. Before participating, subjects signed informed consent to procedures reviewed and approved by an institutional review board. All experimental procedures conformed to the tenets of the Declaration of Helsinki.

\section{EXPERIMENTAL DESIGN}

Patients and controls were each studied on a single occasion. The eye with the more severe visual field defect was chosen in patients; the right eye was examined in controls. Patients ceased all ocular and systemic medications for 3 weeks before study. 

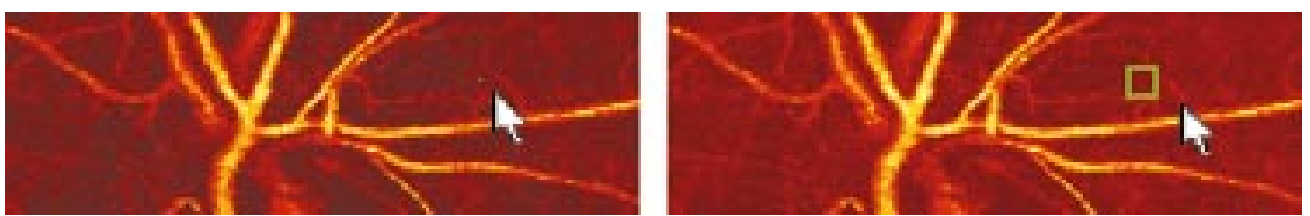

Figure 1 Confocal scanning laser Doppler flowmetry (cSLDF) of peripapillary retina. Left: arrow indicates $1 \times 1$ pixel $(10 \times 10 \mu \mathrm{m})$ measurement window, which, for pixel by pixel analysis, is moved over the entire image for data collection (large vessels and areas with inadequate focus (including rim and cup areas posterior to the focal plane) are excluded). Right: arrow indicates $10 \times 10$ pixel $(100 \times 100 \mu \mathrm{m})$ measurement window for conventional analysis, placed approximately $100 \mu \mathrm{m}$ from the disc margin.

Table 1 Blood flow in $100 \times 100 \mu m$ boxes in normal tension glaucoma (NTG) patients and controls

\begin{tabular}{llll}
\hline Anatomical site & NTG & Control & p Value \\
\hline $\begin{array}{l}\text { Temporal peripapillary } \\
\quad \text { retina }\end{array}$ & $402(48)$ & $402(47)$ & 0.99 \\
Nasal peripapillary retina & $317(43)$ & $345(36)$ & 0.63 \\
Average of above two areas & $363(31)$ & $374(26)$ & 0.80 \\
\hline
\end{tabular}

\section{PROCEDURES}

Examination technique

With the subject's head and chin comfortably secured, he or she fixated on a letter on an acuity chart placed 8 feet behind the operator. When the operator had positioned the camera, the subject fixated on a single portion of a letter until measurements were complete.

\section{Data acquisition}

In video mode, the cSLDF (Heldelberg retinal flowmeter; Heidelberg Engineering; $\mathrm{GmbH}$, Heidelberg, Germany) images a $2560 \times 2560$ $\mu \mathrm{m}$ area of retina or optic nerve head with a scanning $785 \mathrm{~nm}$ diode laser. Green lines on the operator screen mark the boundaries of the $2560 \times 640 \mu \mathrm{m}$ area from which flow data are derived. After this area is scanned, the cSLDF computer performs a fast Fourier transform to extract the individual frequency components of the reflected light. From each $10 \times 10 \mu \mathrm{m}$ pixel in the scan, a power spectrum is calculated: the frequency location on the $\mathrm{x}$ axis represents a blood velocity, while the height of the spectrum at that point represents the number of blood cells required to produce that intensity. Integrating the spectrum yields a value proportional to the total number of red blood cells times their velocity: this "blood flow" is determined in arbitrary units.

\section{ANALYSIS FROM THE INSTRUMENT DEFAULT \\ "SMALL BOX"}

A single sample box $10 \times 10$ pixels in size $(100$ $\times 100 \mu \mathrm{m})$, free from motion artefact and major vessels, is selected within the image. During each measurement session two of these small boxes were chosen for each subject-one each within the temporal and nasal peripapillary retina. When the retinal plane is in focus the neuroretinal rim area is too dark and the

Table 2 Pixel by pixel flow analysis in normal tension glaucoma (NTG) patients and controls

\begin{tabular}{llll}
\hline Aspect of analysis & NTG & Control & p Value \\
\hline $\begin{array}{l}\text { Minimal flow (\% of total pixels) } \\
\text { Flow in selected pixels (arbitrary units) }\end{array}$ & $30.0(3.3)$ & $18.5(1.8)$ & 0.006 \\
25th percentile & $27(13)$ & $79(16)$ & 0.02 \\
50th percentile & $185(38)$ & $324(27)$ & 0.007 \\
75th percentile & $446(64)$ & $636(50)$ & 0.03 \\
90th percentile & $751(100)$ & $1017(89)$ & 0.06 \\
\hline
\end{tabular}

cup is posterior to the focal plane and hence out of focus. A single flow value was generated from each small box, and a mean flow from the two small boxes was determined for each subject. Figure 1 (right) shows typical placement of the $10 \times 10$ pixel box, approximately $100 \mu \mathrm{m}$ from the disc margin.

\section{Analysis from the entire image}

When flow histograms are generated from the entire image, flow is recorded for every pixel within the $256 \times 64$ pixel image. Figure 1 (left) shows the $1 \times 1$ pixel sampling window. After elimination of pixels that contain major vessels, are poorly focused, or are improperly illuminated, the remaining values are sorted on the basis of flow. As with the $10 \times 10$ pixel box, the retinal plane is in focus, while the rim and cup area, posterior to the focal plane, remain very dark and excluded from analysis. For each subject, the number of pixels with less than one arbitrary unit of flow ("minimal flow pixels") is determined as a percentage of total pixels, and the flow in the pixel at the 25 th, 50 th, 75 th, and 90 th percentile of flow is determined.

\section{STATISTICAL ANALYSIS}

Comparisons of values obtained from patients and controls were made using two tailed unpaired $t$ tests, with $\mathrm{p}<0.05$ regarded as significant.

\section{Results}

PATIENTS $V$ CONTROLS: DEFAULT SMALL BOX

ANALYSIS

Patients and controls did not differ in cSLDF measurements of blood flow using the default $10 \times 10$ pixel box in either the temporal or nasal peripapillary retina. In addition, the two groups did not differ in flow when values obtained from these two areas were averaged. Group mean values for blood flow, as measured at each site, and the average from the two sites, are shown in Table 1.

PATIENTS $V$ CONTROLS: ENTIRE IMAGE ANALYSIS Approximately 1200 pixels, obtained from the entire peripapillary retinal image, were included for an average subject in this phase of the study. This number, which did not differ between patients and controls, represents a sample size approximately 12 -fold greater than that obtained from the default $10 \times 10$ pixel box. The percentage of these qualifying pixels that contained minimal flow was significantly increased in NTG patients (Table 2). In addition, flow in the pixels at the 25 th, 50 th, and 
75th percentile of flow were significantly reduced in the patients as compared with controls (Table 2).

\section{Discussion}

In this study we found reduced peripapillary retinal capillary blood flow in normal tension glaucoma (NTG) patients compared with age matched controls, using analysis of the entire confocal scanning laser Doppler flowmeter (cSLDF) image. These reductions presented as increased areas of minimal flow in NTG patients. The preponderance of minimal flow and other low flow areas, compared with the age matched controls, indicates reduced flow within pixels at the 25th, 50th, and 75th percentile flow in NTG patients. These capillary perfusion differences were not detectable using "default" analysis of $10 \times 10$ pixel boxes, as placed within either the nasal or temporal peripapillary retina, or as averaged from these two areas.

The cSLDF was developed to measure capillary perfusion of tissue close to the site of nerve damage in glaucoma patients. ${ }^{913}{ }^{14}$ Our findings directly document blood flow reductions within the peripapillary retina in NTG. These results are consistent with more indirect estimates of ocular perfusion (from indocyanine green angiography, colour Doppler imaging, and whole brain magnetic resonance imaging) suggesting that vascular dysfunction characterises this illness. ${ }^{4-8}$ Recent studies also suggest that primary open angle glaucoma (POAG) patients may suffer from blood flow reductions at the optic nerve head and in the peripapillary retina, further suggesting that blood flow deficits may be a consistent finding in glaucoma, independent of IOP. ${ }^{15} 16$

The cSLDF accurately measures blood flow in an artificial capillary tube $(r=0.97, \mathrm{p}$ $<0.000^{9}$ ), providing results similar to commercially available laser Doppler flowmeters. ${ }^{17}$ The method also displays coefficients of reliability near 0.85 for immediately repeated volume, velocity, and flow measurements from $10 \times 10$ pixel sampling sites. ${ }^{17}$ However, long term reproducibility from these small sampling boxes is less adequate, with the coefficient of variation of measures repeated each week ${ }^{18}$ for 4 weeks averaging $30 \%$ of the mean. ${ }^{12} \mathrm{~A}$ smaller sampling area (for example, a $4 \times 4$ pixel box ${ }^{19}$ ) exacerbates these problems, and is even less reproducible than the larger box. ${ }^{19}$ However, broadening the analysis to include every qualifying pixel within the entire image (in this study, every qualifying pixel within the peripapillary retina) improves test/retest reliability, reducing the coefficient of variation of repeated weekly measurements to $\sim 15 \%$ of the mean for selected portions of the flow histogram. ${ }^{12}$ This increase in sensitivity likely results from a simple increase in data density: even after elimination of data from large vessels and pixels that are inadequately focused, the number of pixels included in entire image analysis in this study was approximately 12-fold higher than that obtained from the default small box. The reduction in coefficient of variation from $30 \%$ to $15 \%$, obtained by shifting from default small box analysis to pixel by pixel analysis, reduces the sample sizes required for a given statistical power by a factor of four. ${ }^{18}$

Consequently, in this study comparing 12 controls with 12 NTG patients, measurements from the default small box detected no differences in blood flow, while histograms generated from the entire image found significant differences between the two groups. Previous studies of peripapillary retinal and optic disc rim flow in glaucoma have found conflicting results, with some authors finding reduced flow in (POAG) patients, ${ }^{16}$ while others find similar levels of perfusion in POAG and NTG patients, and control subjects. ${ }^{10}{ }^{11}$ Although these earlier studies involved relatively large sample sizes (up to 40 in a group ${ }^{1011}{ }^{16}$ ), the use of the default 10 $\times 10$ pixel box suggests that prior conflicting results may arise in part from low reproducibility of this method.

Some earlier work suggests that cSLDF may occasionally detect vessels and blood flow in the choroid. ${ }^{11}$ This inference, drawn from finding focal areas of high blood flow in many glaucoma patients, ${ }^{11}$ could not be supported by the results of this study. Rather, blood flow readings were consistently decreased in NTG patients. Perhaps, in these patients with early stage illness free from severe retinal thinning, sampling depth is insufficient for detection of choroidal perfusion. Of course, these speculations do not rule out the possibility that vascular dysfunction in NTG may also include overall or regional aspects of the choroidal circulation itself. ${ }^{6}$

Although our results directly show reduced peripapillary retinal capillary perfusion in NTG, they do not prove that vascular insufficiency causes pathology in this disease. Rather, while these results find reduced perfusion associated with disease, they cannot distinguish cause from effect. It is possible that factors unrelated to blood flow (for example, mechanical compression that alters transport of neurotrophins ${ }^{20}$ ) accelerate ganglion cell apoptosis, while vascular deficits appear later, ${ }^{120}$ although the normal IOP seen in these patients argues against a purely mechanical causation. Consequently, the possibility remains open that blood flow deficits are at least in part cause, rather than entirely consequence of disease: chronic, low grade ischaemia in the brain does stimulate neural cell apoptosis, ${ }^{2}$ and in vivo models show that induced optic nerve ischaemia leads to glaucomatous optic neuropathy. ${ }^{21}$

In summary, in this study cSLDF entire image analysis detected substantial reductions in capillary perfusion of the peripapillary retina in NTG patients compared with controls. The increase in minimal and low flow regions in these tissues in NTG show that reduced perfusion is certainly a correlate, and possibly a contributing cause, of the retinal ganglion cell death that defines this disease.

Supported in part by NIH grant EY10801 (Dr Harris), by an unrestricted grant from Research to Prevent Blindness, and the CS First Boston Research Fund of the Glaucoma Foundation. 
Dr Harris is the 1995 William and Mary Greve International Research Scholar.

1 Quigley HA, Nickells RW, Kerrigan LA, et al. Retinal ganglion cell death in experimental glaucoma and after ganglion cell death in experimental glaucoma and after 1995;36:774-86.

2 Nickells RW. Retinal ganglion cell death in glaucoma: the how, the why, and the maybe. F Glaucoma 1996;5:345-56.

3 Hernandez MR, Pena JDO. The optic nerve head in glaucomatous optic neuropathy. Arch Ophthalmol 1997;115:38995.

4 Butt Z, O'Brien C, McKillop G, et al. Color Doppler imaging in untreated high- and normal-pressure open-angle glaucoma. Invest Ophthalmol Vis Sci 1997;38:690-6.

5 Kaiser HJ, Schoetzau A, Stümpfig, et al. Blood-flow velocities of the extraocular vessels in patients with high-tension and normal-tension primary open-angle glaucoma. $A m \mathcal{F}$ Ophthalmol 1997;123:320-7.

6 Duijm HFA, van den Berg TJTP, Greve EL. A comparison of retinal and choroidal hemodynamics in patients with primary open-angle glaucoma and normal-pressure glaucoma. Am f Ophthalmol 1997;123:644-56

7 Brart DPS, de Souza Lima M, Bartsch D-U, et al. Indocyanine green angiography of the peripapillary region in glaucomatous eyes by confocal scanning laser ophthalmoscopy. comatous eyes by confocal scanning

8 Stroman GA, Stewart WC, Golnik KC. Magnetic resonance imaging in patients with low-tension glaucoma. Arch Ophthalmol 1995:113:168-72.

9 Michelson G, Langhans M, Groh MJM. Clinical investigation of the combination of a scanning laser ophthalmoscope and laser Doppler flowmeter. Ger f Ophthalmol 1995;4:342-9.

10 Hollo G, van den Berg TJ, Greve EL. Scanning laser Doppler flowmetry in glaucoma. Int Ophthalmol 1996-97;20: 63-70
11 Hollo G, Greve EL, van den Berg TJ, et al. Evaluation of the peripapillary circulation in healthy and glaucomatous eyes with scanning laser Doppler flowmetry. Int Ophthalmol 1996-97;20:71-7.

12 Kagemann L, Harris A, Chung HS, et al. Heidelberg retinal flowmetry: factors affecting blood flow measurement. $\mathrm{Br} \mathcal{F}$ Ophthalmol 1998;82:131-6.

13 Strenn K, Menapace R, Rainer G, et al. Reproducibility and sensitivity of scanning laser Doppler flowmetry during graded changes in $\mathrm{PO}_{2}$. Br f Ophthalmol 1997;81:360-4.

14 Langhans M, Michelson G, Groh MJM. Effect of breathing $100 \%$ oxygen on retinal and optic nerve head capillary blood flow in smokers and non-smokers. Br f Ophthalmol 1997;81:365-9.

15 Michelson G, Langhans MJ, Groh MJM. Perfusion of the juxtapapillary retina and the neuroretinal rim area in primary open angle glaucoma. F Glaucoma 1996;5:91-8.

16 Nicolela MT, Hnik P, Drance SM. Scanning laser Doppler flowmeter study of retinal and optic disk blood flow in glaucomatous patients. Am $\mathcal{F}$ Ophthalmol 1996;122:77583.

17 Michelson G, Schmauss B. Two dimensional mapping of the perfusion of the retina and optic nerve head. $\mathrm{Br} \mathcal{F} \mathrm{Oph}-$ thalmol 1995;79:1126-32.

18 Snedecor GW, Cochran WG. Statistical methods. 7th ed. Ames: Iowa State University Press, 1980:83-106.

19 Nicolela MT, Hnik P, Schulzer M, et al. Reproducibility of retinal and optic nerve head blood flow measurements with scanning laser Doppler flowmetry. F Glaucoma 1997;6: scanning

20 Minckler DS, Bunt AH, Johanson GW. Orthograde and retrograde axoplasmic transport during acute ocular hypertension in the monkey. Invest Ophthalmol Vis Sci 1977;16: $426-41$.

21 Orgül S, Cioffi GA, Wilson DJ, et al. An endothelin-1 induced model of optic nerve ischemia in the rabbit. Invest Ophthalmol Vis Sci 1996;37:1860-9. 